\title{
Endobronchial tuberculosis: histopathological subsets and microbiological results
}

\author{
Sevket Ozkaya ${ }^{*}$, Salih Bilgin², Serhat Findik ${ }^{3}$, Hayriye Çete Kök², Canan Yuksel ${ }^{4}$ and Atilla Güven Atıc ${ }^{5}$
}

\begin{abstract}
Background: Endobronchial tuberculosis (EBTB) is defined as a tuberculous infection of the tracheobronchial tree with microbial and histopathological evidence, with or without parenchymal involvement. Bronchoscopic appearances of EBTB have been divided into seven subtypes: actively caseating, edematous-hyperemic, fibrostenotic, tumorous, granular, ulcerative, and nonspecific bronchitic. However, information for establishing a definite microbiological diagnosis in each of these categories is lacking. We aimed to present bronchoscopic appearances and percentages for the EBTB subtypes and to compare bronchoscopic appearances with microbiological positivity in bronchial lavage fluid.

Methods: From 2003 to 2009, 23 biopsy-proven EBTB patients were enrolled in the study. Diagnosis of EBTB was histopathologically confirmed in all patients.

Results: The commonest subtype was the edematous-hyperemic type (34.7\%); other subtypes in order of occurrence were: tumorous (21.7\%), granular (17.3\%), actively caseating (17.3\%), fibrostenotic (4.3\%), and nonspecific bronchitic (4.3\%). Although all patients were sputum-smear-negative for acid-fast bacilli (AFB), 26\% of patients were smear-positive for AFB in the bronchial lavage fluid. The bronchial lavage fluid grew Mycobacterium tuberculosis in $39.1 \%$ of all patients.

The bronchial lavage smear positivity for AFB in the bronchial lavage fluid was 75\%, 25\%, 20\%, $12.5 \%$, 0\%, and 0\% for the granular, actively caseating, tumorous, edematous-hyperemic, fibrostenotic, and nonspecific bronchitic subtypes of EBTB, respectively. Culture positivity for Mycobacterium tuberculosis in bronchial lavage fluid was 75\%, $50 \%, 40 \%, 25 \%, 0 \%$, and $0 \%$, respectively.

Conclusion: The commonest subtype of EBTB was the edematous-hyperemic subtype. The granular type had the highest smear positivity and culture positivity for Mycobacterium tuberculosis in bronchial lavage fluid. Bronchoscopy should be performed in all patients suspected to have EBTB.
\end{abstract}

Keywords: Bronchoscopy, Endobronchial tuberculosis, Microbiology, Radiology

\section{Background}

Tuberculosis (TB) is a disease of poverty, affecting the most vulnerable groups of the world's population: more than half of TB-related deaths occur in Asia, while the greatest TB burden as percentage of population is in Africa [1]. Endobronchial tuberculosis (EBTB) is defined as a tuberculous infection of the tracheobronchial tree with microbial and histopathological evidence, with or without parenchymal involvement [2]. The incidence of EBTB was reported as $5.88 \%$ in cases with pulmonary

\footnotetext{
* Correspondence: ozkayasevket@yahoo.com

${ }^{1}$ Rize University, Faculty of Medicine, Department of Pulmonary Medicine, Rize, Turkey

Full list of author information is available at the end of the article
}

tuberculosis [3]. However, since bronchoscopy is not routinely performed in patients with tuberculosis, the true incidence of EBTB is likely underestimated. There is only one study in the literature in which bronchoscopy was performed in all patients with active pulmonary tuberculosis, and it reported an incidence of EBTB as high as 50\% [4]. EBTB continues to be a clinical challenge, especially in endemic areas, since it may mimic a variety of pulmonary diseases such as bronchogenic carcinoma, pneumonia, or bronchial asthma. The clinical course of EBTB is rather variable due to the interactions among the effects of mycobacteria, host immunity, and antituberculosis drugs; any variation in these three

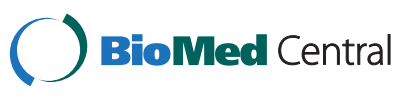


factors may result in different clinical presentations. Chung classified endobronchial tuberculosis into seven subtypes according to bronchoscopic features: actively caseating, fibrostenotic, edematous-hyperemic, tumorous, ulcerative, granular, and nonspecific bronchitic [5]. It was also found that the classifications was valuable to predict the therapeutic outcome of EBTB [3]. However, to our knowledge, there are not enough published data comparing the subtypes of EBTB with microbiological confirmation either using acid-fast bacilli (AFB) stain and/or culture positivity for the organism in the bronchial lavage (BL) fluid. Likewise, we could not find sufficient examples of the bronchoscopic appearances of EBTB subtypes in currently published articles. Therefore, in this study we aimed to present bronchoscopic images and percentages of the EBTB subtypes and to compare bronchoscopic appearances with the microbiological positivity in BL fluid.

\section{Methods}

The Samsun Chest Diseases and Thoracic Surgery Hospital is a reference hospital for tuberculosis in Turkey. From 2003 to 2009, 23 biopsy-proven EBTB patients were enrolled in this study. Diagnosis of EBTB was confirmed histopathologically in all patients. Patient data were gathered and evaluated retrospectively. All patients were sputum-smear-negative according to AFB staining, and fiberoptic bronchoscopy was performed in the case of suspected tuberculosis or for the differential diagnosis of tuberculosis. Premedication for the bronchoscopy was done with intramuscular atropine at $0.01 \mathrm{mg} / \mathrm{kg}$ and opioids. The pharynx and vocal cords were also anesthetized with nebulized lidocaine. A flexible bronchoscope was inserted through the nasal passage. After inspection of the airways and before the biopsies, the bronchoscope was wedged into the airway. Sterile Saline, in aliquots of 50-60 mL, was delivered via syringe into the distal airway through the suction channel of the bronchoscope. It was then quickly aspirated through a gentle suction into a collection trap. Forceps were advanced through the bronchoscope and airway to obtain biopsies from bronchial lesions (EBBx). The patients' characteristics, including demographic data, symptoms, initial diagnoses, and radiological, bronchoscopic and microbiological features, were reviewed and evaluated retrospectively. Bronchoscopic findings were categorized according to Chung's classifications [4]. The results are presented as mean and percentage of patients. The study was approved by the institutional review board and written consent to use their medical records was obtained from the patients.

\section{Results and discussion}

During the study period a total of 1,064 patients were diagnosed with pulmonary tuberculosis at our hospital.
23 patients out of them, who were histopathologically diagnosed with EBTB via $\mathrm{EBBx}$, form the study group. The incidence of EBTB was $2.2 \%$. Demographic and clinical features are given in Table 1. The mean age of the patients was 39.6 years (range: 15-72 yrs). The female-to-male ratio was nearly 1 . The commonest symptom was cough (86.9\%), followed by constitutional symptoms (52.1\%), dyspnea (39.1\%), sputum (34.7\%), fever (30.4\%), and hemoptysis (13\%). Initial working diagnoses were lung cancer $(47.8 \%)$, communityacquired pneumonia (34.7\%), and tuberculosis (17.3\%). At the radiographic examination lesions were mainly located in the right lung (87\%), and the commonest radiologic features were consolidation $(47.8 \%)$ and mass lesion (34.7\%) (Figure 1). Cavitation and pleural effusion were seen in $17.3 \%$ of patients (Figure 2).

Bronchoscopic findings were categorized according to Chung's classifications and the subtypes were: edematoushyperemic (34.7\%), tumorous (21.7\%), granular (17.3\%), actively caseating (17.3\%), fibrostenotic $(4.3 \%)$, and nonspecific bronchitic (4.3\%) (Figures 3, 4, 5, 6). No cases of the ulcerative subtype were seen in our patients (Table 2).

Anatomically, the bronchoscopic findings were primarily located in the right upper lobe bronchus with an incidence of $47.8 \%$, which was followed by the middle lobe bronchus (21.7\%) and the right lower lobe bronchus and the left upper bronchus (13\%).

Microbiologic and smear examination of BL fluid was positive for AFB in $26 \%$ of the patients. The highest smear positivity for AFB was found in granular-type cases (75\%). Cultures of BL fluids for Mycobacterium tuberculosis were positive in $39.1 \%$ of patients, and the positivity was highest in granular-type cases $(75 \%)$. The

Table 1 Baseline characteristics of the patients

\begin{tabular}{ll}
\hline Characteristics & $\mathbf{n}(\%)$ \\
\hline Mean age, years (range) & $39.6(15-72)$ \\
\hline$<45$ years & $10(43.4)$ \\
\hline Female/Male & $12 / 11$ \\
\hline Symptoms & $20(86.9)$ \\
\hline Cough & $8(34.7)$ \\
\hline Sputum & $9(39.1)$ \\
\hline Dyspnea & $3(13)$ \\
\hline Hemoptysis & $7(30.4)$ \\
\hline Fever & $12(52.1)$ \\
\hline Constitutional sypmtoms* & \\
\hline Initial Diagnoses & $11(47.8)$ \\
\hline Lung Cancer & $8(34.7)$ \\
\hline Pneumonia & $4(17.3)$ \\
\hline Tuberculosis
\end{tabular}

* Constitutional symptoms; weight loss, weakness, anorexia. 


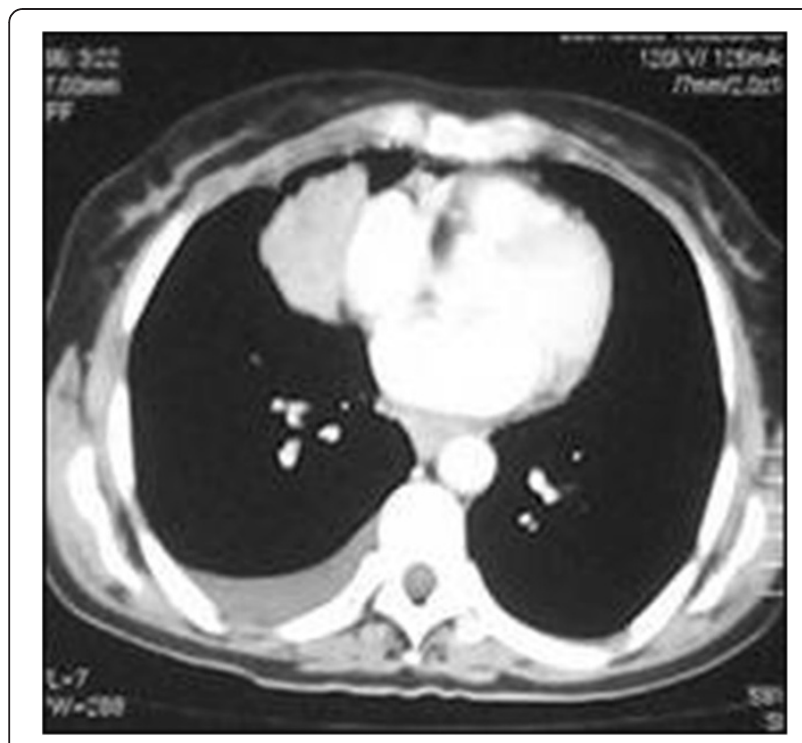

Figure $1 \mathrm{CT}$ showing a right paracardiac intrapulmonary mass lesion and pleural effusion.

results of smears and cultures for Mycobacterium tuberculosis are shown in Table 2.

The histopathologic examination of bronchoscopic biopsies revealed caseating granulomatous inflammation in all patients.

The present study classifies EBTB cases according to Chung's classification and investigates whether there are any differences in microbiologic results among these subtypes for AFB staining and tuberculosis culture. The edematous-hyperemic subtype was the commonest appearance, seen nearly in one-third of patients. While the highest smear and culture positivities for Mycobacterium tuberculosis in BL fluid were seen in the granular type, both tests were negative in patients with fibrostenotic and nonspecific bronchitic EBTB.

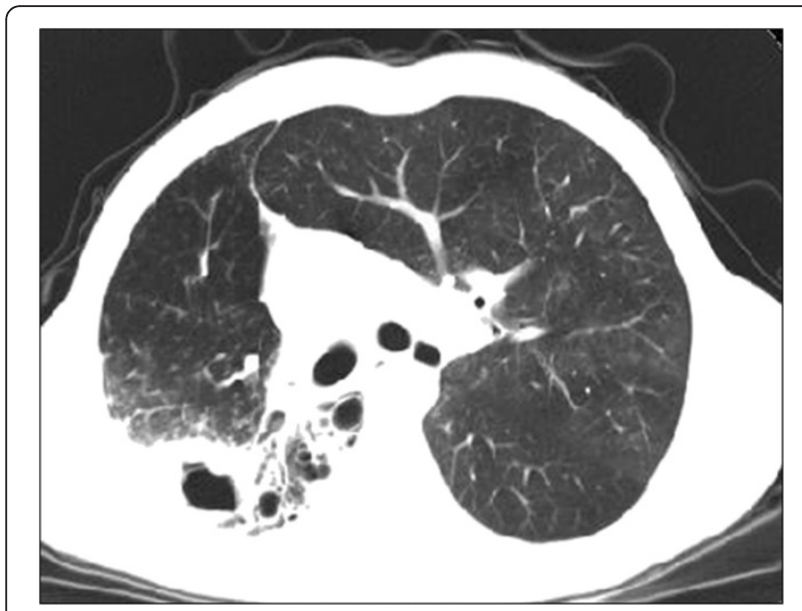

Figure 2 CT showing cavitation and infiltration.

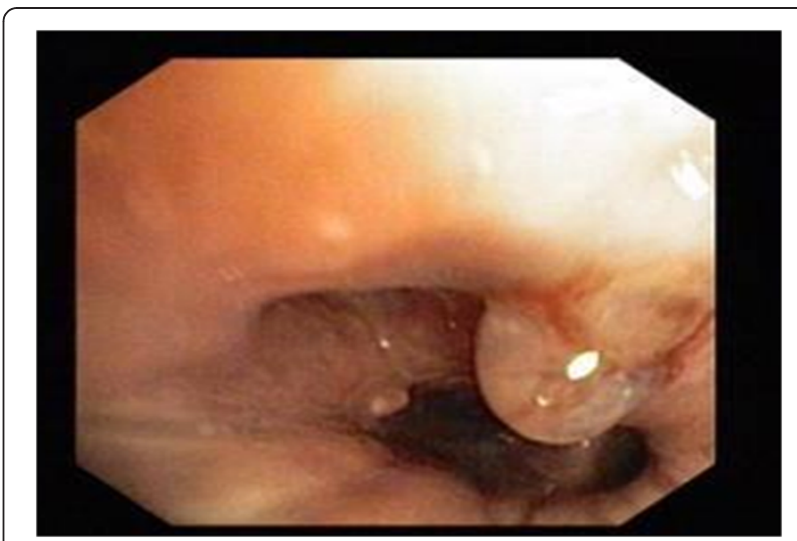

Figure 3 Videobronchoscopy image showing the granuler subtype of endobronchial tuberculosis.

The incidence of EBTB in the present study was $2.2 \%$ in all pulmonary TB patients. This incidence may not reflect the real situation because the retrospective analysis was based on passive detection. The incidence was lower because bronchoscopy is not routinely performed in all patients with TB; the actual incidence of EBTB remains underestimated [3]. EBTB usually affects adults, including both younger and elderly patients ranging 14 to 81 years [6]. In our study the mean age was 39.6 years and $38 \%$ of patients were younger than 45 years, which was consistent with the literature. The female-to-male ratio was 12:11; EBTB was more frequent in female patients (5:1 in females and 2:1 in males). One of the possible reasons for female predominance in our region may be the fact that women usually do not expectorate sputum [5]. The commonest symptom was cough, which was consistent with previous studies.

The pathogenesis of EBTB is not fully known yet. However, possible sources of EBTB may include direct implantation of tubercle bacilli into the bronchus from an adjacent parenchymal lesion, or from an adjacent tuberculous mediastinal lymph node, hematogenous spread, erosion of an intraparenchymal tuberculous lymph node into the bronchus, or extension to the peribronchial region by lymphatic drainage [7-10].

The clinical course of EBTB is quite variable because the interactions among the effects of mycobacteria, host immunity, and antituberculosis treatment is complex; any variation in these factors may determine a change in the course of the disease $[6,11]$. With respect to pathology, EBTB lesions can be divided into six steps: 1 ) The initial lesion presents as simple erythema and edema of the bronchial mucosa with lymphocytic infiltration of the submucosa [12]. 2) Submucosal tubercle formation produces the erythema and granularity seen during bronchoscopy, and stenosis of the bronchial lumen is due to congestion and edema [13]. 3) Development of 


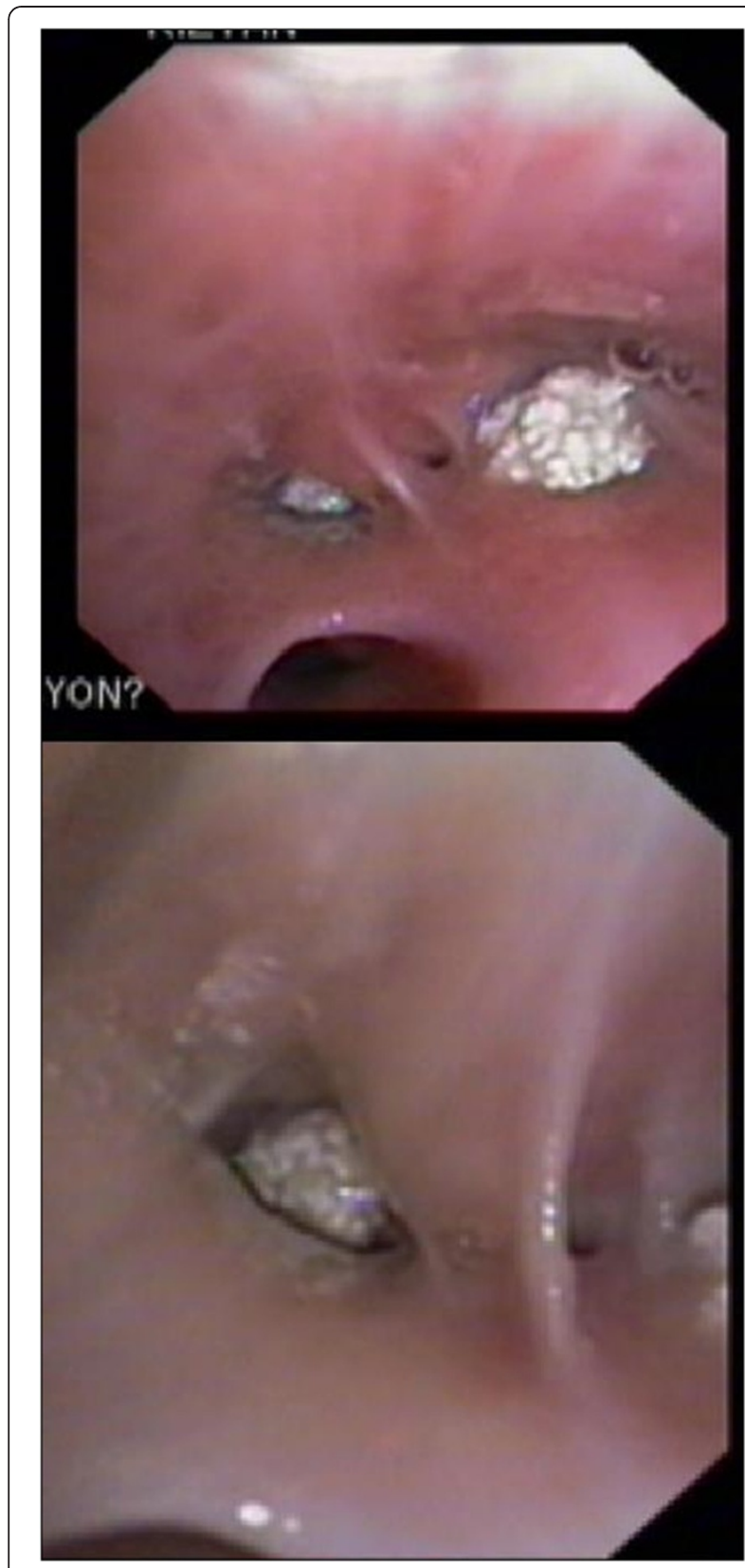

Figure 4 Videobronchoscopy image showing the tumorous subtype of endobronchial tuberculosis.

caseous necrosis with formation of tuberculous granuloma can be found at the mucosal surface [13]. 4) When the inflammation erupts through the mucosa, an ulcer is seen. 5) The ulcer of bronchial mucosa evolves into hyperplastic inflammatory polyps. 6) The endobronchial tuberculous lesion heals by fibrostenosis [7]. Chung's classification was based on this six-step pathological course of tuberculosis, respectively represented by the 1) nonspecific bronchitic, 2) granular and edematoushyperemic, 3) actively caseating, 4) ulcerative, 5) tumorous, and 6) fibrostenotic types [4].

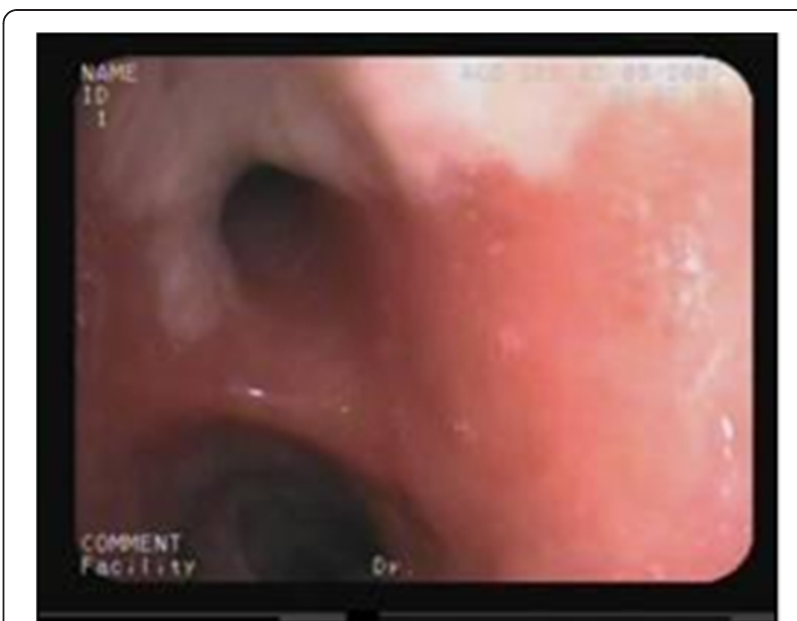

Figure 5 Videobronchoscopy image showing the actively caseating subtype of endobronchial tuberculosis.

The clinical course of EBTB reflects interdependent interactions among many factors because the bronchial inflammatory reactions vary for each patient. Since the clinical course of EBTB is quite variable, all patients suspected of EBTB should be subjected to sputum smear tests and culture examination for tuberculosis [6].

The yield of sputum smear for AFB is not as high as it is in parenchymal involvement, even in an optimal laboratory set up for meticulous sputum examination. In recent studies, sputum positivity in EBTB has been demonstrated to range from $16 \%$ to $53.3 \%[5,14,15]$. In our study, sputum smears for AFB were negative in all patients. The low yield of sputum AFB smears may be

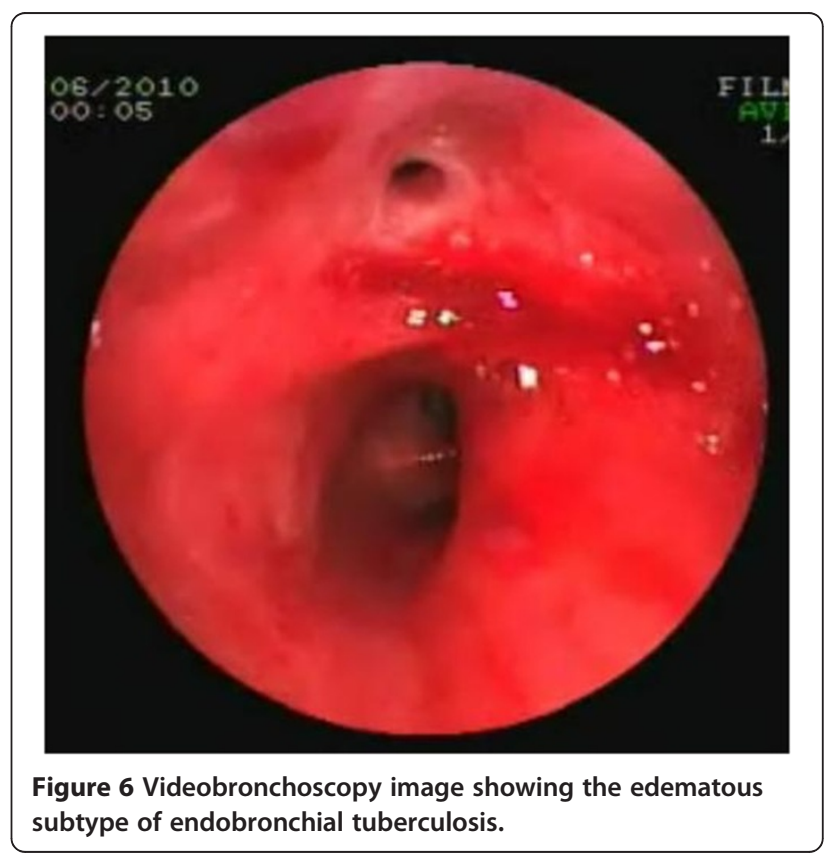


Table 2 Bronchoscopic, histopathologic and microbiologic features of patients

\begin{tabular}{|c|c|c|c|c|}
\hline \multirow[t]{2}{*}{ Types } & \multirow[t]{2}{*}{ n (\%) } & \multirow[t]{2}{*}{ Histopathology of bronchial biopsy } & \multicolumn{2}{|c|}{ BL positivity AFB stain Culture } \\
\hline & & & n (\%) & n (\%) \\
\hline Edematous,hyperemic & $8(34.7)$ & Caseating Granulomatous Inflammation & $1(12.5)$ & $2(25)$ \\
\hline Tumorous & $5(21.7)$ & Caseating Granulomatous Inflammation & $1(20)$ & $2(40)$ \\
\hline Granular & $4(17.3)$ & Caseating Granulomatous Inflammation & $3(75)$ & $3(75)$ \\
\hline Caseating & $4(17.3)$ & Caseating Granulomatous Inflammation & $1(25)$ & $2(50)$ \\
\hline Fibrostenotic & $1(4.3)$ & Caseating Granulomatous Inflammation & 0 & 0 \\
\hline Nonspecific Bronchitic & $1(4.3)$ & Caseating Granulomatous Inflammation & 0 & 0 \\
\hline Total & 23 & & $6(26.1)$ & $9(39.1)$ \\
\hline
\end{tabular}

Table 1 legend - AFB, Acid-fast bacilli; BL, Bronchial Lavage.

due to mucus entrapment by proximal granulation tissue. Therefore, a negative sputum smear does not preclude the diagnosis of EBTB.

The second step in clinical evaluation was bronchoscopy, to examine bronchial structures and obtain specimens for diagnosis. Different bronchoscopic specimens, including biopsy samples, brushings, and washings, may be obtained [6]. A bronchoscopic biopsy is the most reliable method for diagnosing EBTB, because needle aspiration can provide only a cytological diagnosis. Bronchial biopsies may be positive in $30 \%$ to $84 \%$ of patients $[14,16]$. In a clinical analysis of 90 cases of EBTB in China, bronchial brushings yielded variable results, ranging from $10 \%$ to $85 \%$ [14].

To the best of our knowledge, there has been no study to date comparing the microbiologic results with the different features of EBTB. BL fluid was positive for AFB in $26 \%$ of the cases and culture positivity for TB bacilli in the fluid was positive in $39.1 \%$. The highest positivity for AFB was found in the granular type of EBTB (75\%). The highest positivity for mycobacterial culture was also found in the granular type (75\%). It is thus clear that microbiologic methods were more often found to be positive for early lesions, namely in the granular and actively caseating subtypes of EBTB. We think that the granular type of EBTB may reflect the earliest granulomatous inflammatory reaction against Mycobacterium tuberculosis.

Since microbiologic methods were less positive in some cases, even $0 \%$ at the late fibrostenotic stage, histopathologic methods seem much more important in these cases and endobronchial biopsy is crucial for reaching a final diagnosis.

In this study, there was no difference regarding the sex of the enrolled patients, in fact the female-to-male ratio was nearly 1 , differently from prior studies that showed a marked preponderance of female patients. This may be due to race or ethnicity, as all studies reporting female preponderance were from Korea and Japan [4,5,9].

There are some limitations to our study. First, because the study population was not very large, statistical methods could not be applied for comparison among subtypes. Nevertheless, general proportions allowed for comparison, $75 \%$ positivity for the granular subtype versus $0 \%$ for the fibrostenotic and nonspecific bronchitic subtypes. Second, since this was a retrospective study, some data might have been lost.

\section{Conclusions}

In conclusion, this study showed that in the early granular and actively caseating subtypes of EBTB, the yield of microbiologic methods was higher than in the late tumorous and fibrostenotic subtypes. Therefore, bronchoscopic biopsy including mucosal and parenchymal examination should be added to bronchial lavage and/or brushing in order to increase diagnostic yield in patients suspected of EBTB.

\section{Competing interests}

The authors declare that they have no competing interests.

\section{Author details}

${ }^{1}$ Rize University, Faculty of Medicine, Department of Pulmonary Medicine, Rize, Turkey. ${ }^{2}$ Samsun Chest Disease and Chest Surgery Hospital, Samsun, Turkey. ${ }^{3}$ Ondokuz Mayis University, Faculty of Medicine, Department of Pulmonary Medicine, Samsun, Turkey. ${ }^{4}$ Samsun Pathology and Cytology Centre, Samsun, Turkey. ${ }^{5}$ Ondokuz Mayis University, Faculty of Medicine, Department of Pulmonary Medicine, Samsun, Turkey.

Received: 18 October 2012 Accepted: 18 October 2012

Published: 22 October 2012

\section{References}

1. Casali L, Crapa ME: Women, immigration, poverty and tuberculosis. Multidiscip Resp Med 2010, 5:398-400.

2. Hohisel G, Chan BK, Chan CH, Chan KS, Teschler H, Costabel U: Endobronchial tuberculosis: diagnostic features and therapeutic outcome. Respir Med 1994, 88:593-597.

3. Chung HS, Lee JH: Bronchoscopic assessment of the evolution of endobronchial tuberculosis. Chest 2000, 117:385-392.

4. Kurasawa T, Kuze F, Kawai M, Amitani R, Murayama T, Tanaka E, Suzuki K, Kubo Y, Matsui Y, Sato A, et al: Diagnosis and management of endobronchial tuberculosis. Intern Med 1992, 31:593-598.

5. Chung HS, Lee JH, Han SK, Shim YS, Kim KY, Han YC, et al: Classification of endobronchial tuberculosis by the bronchoscopic features. Tuberc Respir Dis 1991, 38:108-115.

6. Kashyap S, Mohapatra PR, Saini V: Endobronchial tuberculosis. Indian J Chest Dis Allied Sci 2003, 45:247-256.

7. Matthews JI, Matarese SL, Carpenter JL: Endobronchial tuberculosis simulating lung cancer. Chest 1984, 86:642-644. 
8. Smith LS, Schillaci RF, Sarlin RF: Endobronchial tuberculosis. serial fiberoptic bronchoscopy and natural history. Chest 1987, 91:644-647.

9. Lee JH, Park SS, Lee DH, Shin DH, Yang SC, Yoo BM: Endobronchial tuberculosis: clinical and bronchoscopic features in 121 cases. Chest 1992, 102:990-994.

10. Kim YH, Kim HT, Lee KS, Uh ST, Cung YT, Park CS: Serial fiberoptic bronchoscopic observations of endobronchial tuberculosis before and early after antituberculous chemotherapy. Chest 1993, 103:673-677.

11. Chan HS, Pang JA: Effect of corticosteroids on deterioration of endobronchial tuberculosis during chemotherapy. Chest 1989, 96:1195-1196.

12. Shulutko ML, Kazak TI, Tarasov AS: Tuberculosis. In Bronchology. Edited by Lukomsky GI. St Louis, MO: Mosby; 1979:287-305.

13. Medlar EM: The behavior of pulmonary tuberculous lesion: a pathological study. Am Rev Tuberc 1955, 71:132-145.

14. Yu W, Rong Z: Clinical analysis of 90 cases with endobronchial tuberculosis. Zhonghua Jie He He Hu Xi Za Zhi 1999, 22:396-398.

15. Aggarwal AN, Gupta D, Joshi K, Behera D, Jintal SK: Endobronchial involvement in tuberculosis: a report of 24 cases diagnosed by fibreoptic bronchoscopy. J Bronchol Intervent Pulmonol 1999, 6:247-250.

16. Altin S, Cikrikcioglu S, Morgül M, Koşar F, Ozyurt H: 50 endobronchial tuberculosis cases based on bronchoscopic diagnosis. Respiration 1997, 64:162-164.

doi:10.1186/2049-6958-7-34

Cite this article as: Ozkaya et al:: Endobronchial tuberculosis:

histopathological subsets and microbiological results. Multidisciplinary Respiratory Medicine 2012 7:34.

\section{Submit your next manuscript to BioMed Central and take full advantage of:}

- Convenient online submission

- Thorough peer review

- No space constraints or color figure charges

- Immediate publication on acceptance

- Inclusion in PubMed, CAS, Scopus and Google Scholar

- Research which is freely available for redistribution 